\title{
Expression of interleukin-17 in lesions of erythema multiforme may indicate a role for $T$ helper 17 cells
}

\author{
ZEYNEP MELTEM AKKURT $T^{1}$ DERYA UÇMAK', GÜL TÜRKC $\ddot{U}^{2}$, HATICE YÜKSEL ${ }^{3}$, \\ KENAN YILDIZ ${ }^{4}$ MUSTAFA ARICA ${ }^{l}$ \\ ${ }^{1}$ Department of Dermatology, Dicle University Faculty of Medicine, Diyarbakir, Turkey \\ ${ }^{2}$ Department of Pathology, Dicle University Faculty of Medicine, Diyarbakir, Turkey \\ ${ }^{3}$ Department of Biochemistry, Dicle University Faculty of Medicine, Diyarbakir, Turkey \\ ${ }^{4}$ Diyarbakir Training and Research Hospital, Diyarbakir, Turkey
}

\begin{abstract}
Introduction: The aim of this study was to evaluate levels of interleukin (IL)-2, IL-6, IL-8, IL-10, $I L-17 A$ and interferon $\gamma(I F N-\gamma)$ in the serum of patients with erythema multiforme (EM) and to search for the presence of IL-17-expressing cells in lesional samples of EM.

Material and methods: A total of 32 patients (22 females and 10 males) diagnosed with EM of the minor or major type were included in the study. Levels of IL-2, IL-6,IL-8,IL-10, IL-17A and IFN- $\gamma$ in the serum were determined and compared with healthy controls. Biopsy specimens were stained with haematoxylin and eosin (HE) and monoclonal antibodies to CD4, CD8 and IL-17 for immunohistochemical examination.

Results: IL-2, 6, 8 and 17A were significantly higher in the patient group $(p=0.016, p=0.001$, $p=0.004, p=0.006$, respectively) and levels of IL-10 were significantly lower than in the control group $(p=0.046)$. The cellular infiltrate in lesions of EM was composed mainly of CD4+ T lymphocytes. The presence of IL-17-expressing cells, at proportion of 5 to 50\%, was observed in the infiltrate.

Conclusions: The demonstration of IL-17-expressing cells in lesions of EM in this study has brought forth the assumption that Th17 cells may be involved in the pathogenesis of EM.
\end{abstract}

Key words: erythema multiforme, Thelper 17, Thelper 1, interleukin-17.

(Centr Eur J Immunol 2014; 39 (3): 370-376)

\section{Introduction}

Erythema multiforme (EM) is an acute self-limiting mucocutaneous disorder thought to be a reaction to an infection or drug. It is characterized by a polymorphous eruption [1]. The classic presentation is named EM minor and is often preceded by herpes infections [2]. Histopathological findings of EM include liquefactive degeneration of the basal epidermal cells, necrotic keratinocytes, and lymphocyte exocytosis. Subepidermal clefts and vesiculation may develop secondary to extensive basal cell vacuolar degeneration in some cases. A lichenoid pattern consisting of a mild to moderate lymphohistiocytic infiltrate may be seen at the dermo-epidermal junction. A dermal infiltrate typically shows lymphohistiocytic infiltrate surrounding the superficial and mid-dermal vessels. Numerous apoptotic keratinocytes are seen scattered at various levels throughout the epidermis. Erythema multiforme is consid- ered a prototype of interface dermatitis and a model lesion for acute inflammation with mononuclear cells [3-5].

Erythema multiforme has been established as a disease caused by lymphocytes as the predominant effector cells. The number of CD8+ cells have been described to be more numerous in the epidermis, whereas the number of CD4+ cells have been described to be more numerous in the dermis. An increase in the number of Langerhans cells has also been reported [6]. The generation of auto-reactive $T$ cells that target the epidermis plays an important role in the pathogenesis of EM. The stimulus in this process is thought to be the expression of HSV DNA fragments in the skin [7]. Presumably, viral DNA and proteins ingested by macrophages at HSV lesion sites undergo fragmentation and processing for presentation to T cells with HSV memory. HSV DNA encoding the viral DNA polymerase (Pol) is deposited on the skin by circulating CD34+ hematopoietic progenitor cells [8], which transport it from the site of the original HSV

Correspondence: Zeynep Meltem Akkurt, Dicle Üniversitesi Tip Fak Dermatoloji AD Sur, 21280 Diyarbakir, Turkey,

e-mail: meltem@doctor.com 
lesion, and then it is expressed. Activated T cells are recruited to the skin at sites of Pol expression, directly or indirectly resulting in the generation of an inflammatory cascade [9]. These are mainly CD4+ T cells characterized by a T helper type 1 (Th1) response, which promotes the expression of IFN- $\gamma$ and transforming growth factor $\beta$ (TGF- $\beta$ ) $[10,11]$. Erythema multiforme, to a lesser extent, may be drug-induced. Although clinically similar, histopathologically, the inflammatory response in this scenario consists primarily of non-T and CD8+ T cells [6] and the expression of tumor necrosis factor $\alpha$ (TNF- $\alpha)$ [10].

Since the description of a distinct subset of $\mathrm{T}$ cells, $\mathrm{T}$ helper type 17 lymphocytes (Th17), substantial evidence has emerged on their involvement in various autoimmune diseases.

In this study, we aimed to determine the serum cytokine profile of patients with EM. We assumed the presence of a Th1 cytokine profile but in addition we aimed to determine serum IL-17A levels and search for the presence of IL-17A-secreting cells in lesional samples.

\section{Material and methods}

\section{Patients}

This study was conducted at the Dicle University Faculty of Medicine and Diyarbakir Training and Research Hospital Dermatology clinics on patients diagnosed with erythema multiforme, either of the minor or the major subtype, from March 2012 to January 2013. The patients' lesions were biopsied and blood samples were obtained from the patients and from healthy control subjects. Informed consent was obtained from the subjects participating in the study. Approval of the Ethics Committee of Dicle University was obtained and the study was performed in accordance with the ethical standards laid down in the 1964 Declaration of Helsinki and its later amendments. Erythema multiforme with mucosal involvement was classified as erythema multiforme major and in the absence of mucosal disease, the disease was classified as EM minor [3]. All patients had active disease at the time of sample collection.

\section{Blood samples}

Venous blood samples were obtained and immediately centrifuged. Serums were kept at $-80^{\circ} \mathrm{C}$ until laboratory testing. The serum interleukin (IL)-2, IL-6, IL-8, interferon $\gamma$ (IFN- $\gamma$ ) (Invitrogen, Camarillo, CA), IL-10 (Invitrogen, Frederick, MD, CA) and IL-17A (Biosource Europe S. A. Belgium) levels were determined using the enzyme-linked immunosorbent assay method according to the manufacturer's protocols. Serum samples from 29 age- and sex-matched healthy controls were obtained and used for comparison.

\section{Histopathology}

Biopsy samples were obtained using $4 \mathrm{~mm}$ punch biopsy blades. The non-necrotic peripheral areas of the lesions were sampled, avoiding the central necrotic or bullous parts of the lesions.

The punch biopsy materials were fixed in $10 \%$ formalin solution for 24 hours. After biopsy material from each patient was processed by routine histological tissue preparation, all specimens were embedded in wax. $4 \mu \mathrm{m}$ sections of tissue were transferred from paraffin-embedded blocks to four positively charged slides one of which was stained with HE. The other three were stained with CD4 (sc-7219, Santa Cruz Biotechnology), CD8 (C8/144B, Cell Marque) and IL-17 (sc-7927, Santa Cruz Biotechnology) antibodies using an automated immunohistochemical staining device (Ventana Benchmark XT). Samples of human tonsillar tissue and normal skin were used as positive and negative controls, respectively. All samples were reviewed by the same pathologist using a light microscope (Nicon Eclipse 80i) and the results were reported as the percentage of stained cells among the inflammatory infiltrate.

\section{Statistical methods}

Statistical analyses were performed using SPSS 15.0. Mann Whitney U test was used for the analysis of the data. Correlation analyses were done using Spearman's correlation analysis. A $p$ value $<0.05$ was considered to be significant.

\section{Results}

\section{Patients}

A total of 32 patients (22 females and 10 males) diagnosed with EM of the minor or major type were included in the study. 29 patients had EM minor and three patients had EM major. The patients' mean age was $30.56 \pm 2.59$ (range 14-72). Blood samples were obtained from 23 patients and biopsy samples were obtained from 30 patients.

The mean duration of the lesions was 5.73 days. Eleven patients reported that this was their first attack. The rest had had previous attacks. Twenty-two patients had a preceding attack of herpes infection of mean 12.91 days ago. A mean duration of 7.95 days had passed from the onset of herpes to the onset of EM. Five patients reported sun exposure before the attack of EM. Two patients linked their lesions to the preceding upper respiratory tract infection and use of medicine. The three patients with EM major and two patients with EM minor were treated with systemic steroids. The rest were treated with topical steroids.

\section{Blood samples}

The mean age of the patients who donated blood samples was $30.59 \pm 12.40$ (range 14-62).

The controls' mean age was $29.97 \pm 2.43$ (range 18-70) and the difference between the two groups was not statistically significant $(p=0.561)$. The patient group consisted 
Table 1. Mean values of the measured cytokines (mean $\pm \mathrm{SD}, \mathrm{pg} / \mathrm{ml}$ )

\begin{tabular}{lcccccc} 
Parameter & IL-2 & IL-6 & IL-8 & IL-10 & IL-17A & IFN- $\gamma$ \\
\hline Patients & $13.65 \pm 3.00$ & $28.29 \pm 6.81$ & $2.05 \pm 0.36$ & $100.43 \pm 48.54$ & $49.96 \pm 13.43$ & $12.95 \pm 3.44$ \\
\hline Controls & $11.97 \pm 2.73$ & $25.76 \pm 3.72$ & $1.82 \pm 0.19$ & $126.93 \pm 49.96$ & $42.76 \pm 19.14$ & $11.99 \pm 2.65$ \\
\hline $\boldsymbol{p}$ & 0.016 & 0.001 & 0.004 & 0.046 & 0.006 & 0.214 \\
\hline
\end{tabular}

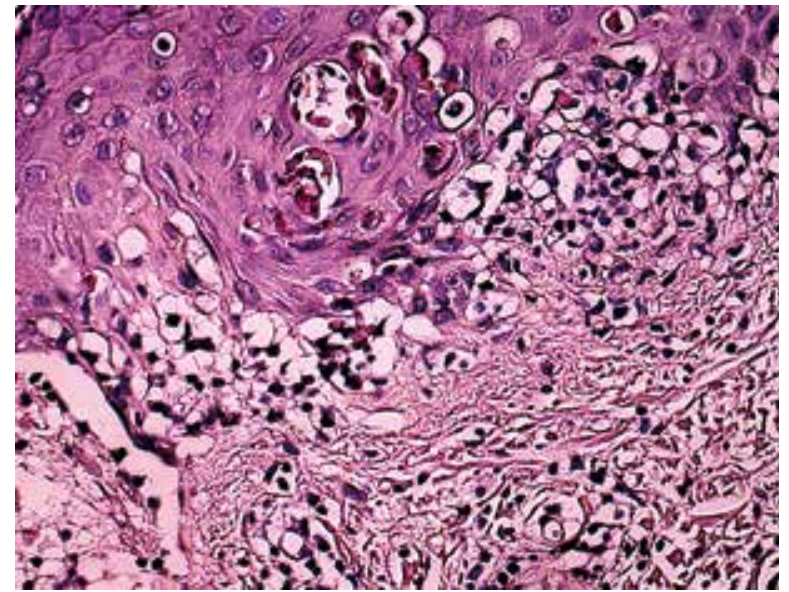

Fig. 1. Basal cell vacuolization and apoptotic keratinocytes in the epidermis with moderate lymphocytic infiltration in the superficial dermis (HE, magnification 200x)

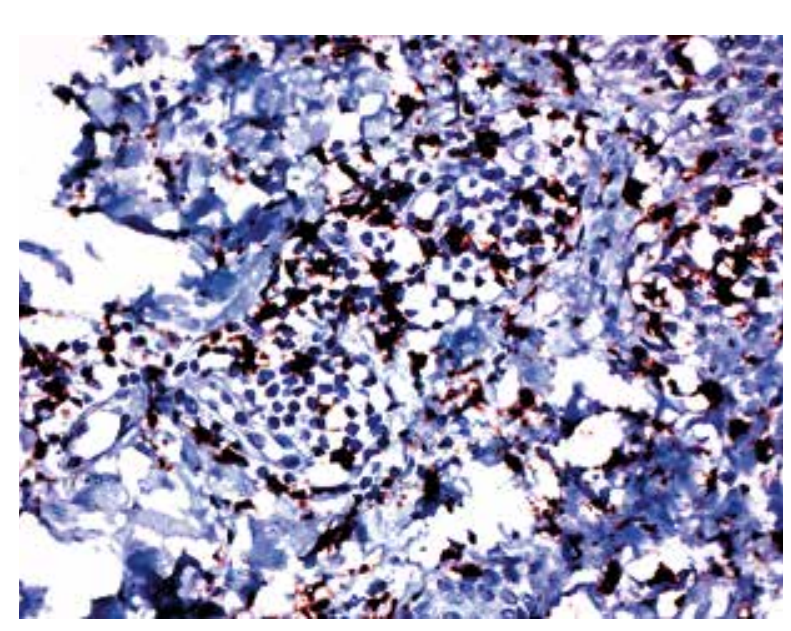

Fig. 3. Staining with CD8 is seen in fewer lymphocytes (magnification 200x)

of 15 females and eight males and the control group consisted of 20 females and nine males.

Mean cytokine values of the patients and controls and $\mathrm{p}$ values are shown in the Table 1. Levels of IL-2, IL-6, IL-8, and IL-17A were significantly higher and IL-10 was significantly lower in the patient group. Levels of IFN- $\gamma$ were not different among the patients and the controls. There was an inverse correlation between levels of IL-17A and IFN- $\gamma(r=-0.413$ and $p=0.045)$ and IL-10 and IFN- $\gamma$

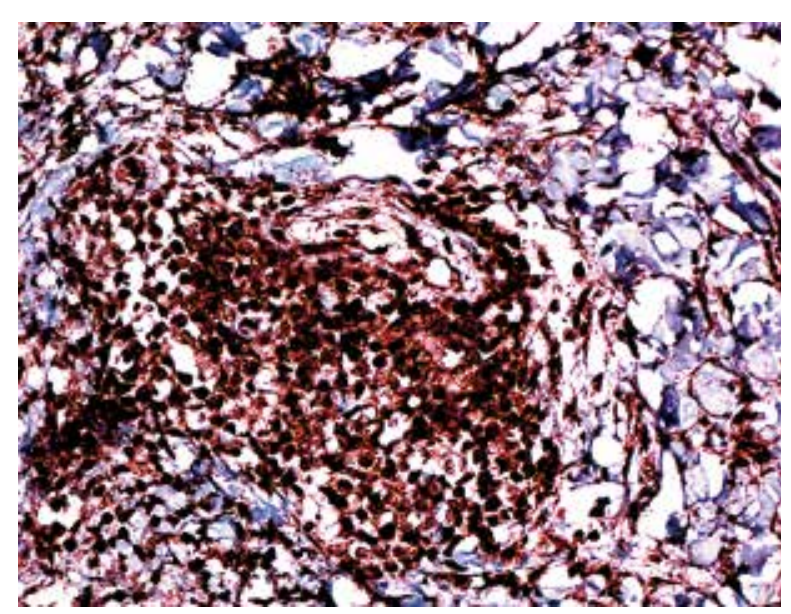

Fig. 2. A majority of lymphocytes are stained with CD4 (magnification 200x)

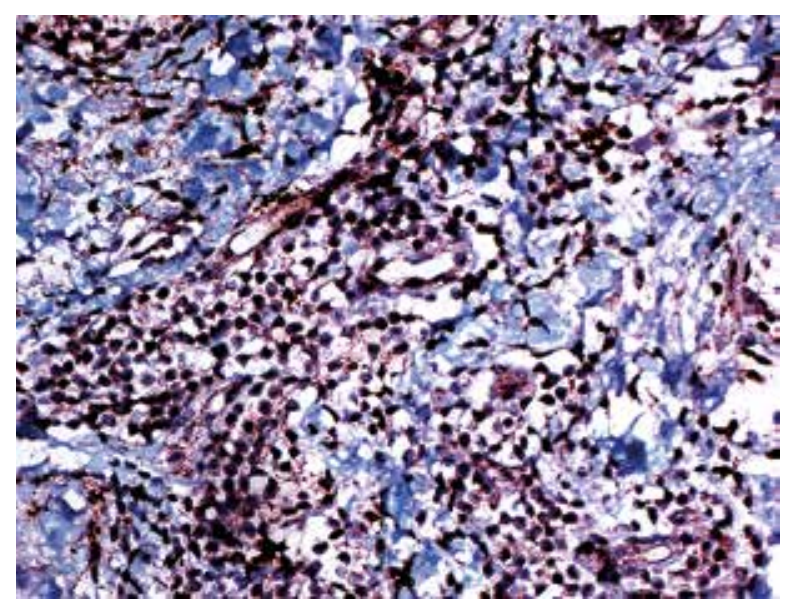

Fig. 4. IL-17 expression is seen among cells of the dermal infiltrate (magnification 200x)

( $r=-0.511$ and $p=0.013$ ) in the patient group. There was an inverse correlation between duration of the lesions and levels of IL-10 ( $r=-0.441$ and $p=0.045)$. Levels of the measured cytokines were not significantly different between patients with and without recurrent lesions.

\section{Histopathology}

Hematoxylin and eosin staining demonstrated typical histopathologic findings of EM in all specimens (Fig. 1). 
The most frequently observed findings were (in a decreasing order) the presence of a lichenoid reaction, vacuolar changes, lymphocyte infiltration in the superficial dermis, epidermal lymphocytes, subepidermal clefts and apoptotic cells. There was a band-like and perivascular infiltration of lymphocytes in the superficial dermis.

On IHC, $50-90 \%$ (mean 73.5\%) of the lymphocytes in the samples were stained with CD4, 10-50\% (mean 26.5\%) were stained with CD8. Interleukin 17-expressing cells were observed in all samples, with their proportions in the infiltrate being 5-15\% (with the exception of one case with $50 \%$ staining, mean 9.33\%) (Figs. 2-4).

\section{Discussion}

The results of this study indicate that levels of IL-2, 6, 8 and 17A were significantly higher in the patient group compared to the control group and levels of IL-10 were significantly lower than in the control group. The presence of a CD4+ T lymphocyte predominance in lesional tissue was observed. In addition, 5 to $50 \%$ of cells in the infiltrate were found to express IL-17.

Increased serum IL-2 is in agreement with the expected Th1 cytokine profile in EM. IL-2 promotes the proliferation of T cells and is among the main Th1 cytokines [12]. It has previously been demonstrated to be increased in the serum of patients with EM [13]. Interferon $\gamma$, one of the major Th1 cytokines, was not elevated in the sera of the patient group in our study. IL-10 is a cytokine involved in the development of regulatory $\mathrm{T}$ cells and also is an effector of Th2 responses [12]. One study has reported that its levels were increased in EM [14] but our results have turned out the opposite. The increased serum IL- 6 and IL- 8 levels lead us to think that the localized lesions of EM may be accompanied by a systemic reaction involving a whole array of cytokines, besides those of the Th1 type. Patients with EM minor predominated our patient profile, with the exception of three who had EM major. Statistically, it was not possible to make a comparison between these two types, but the results of the patients were again significant compared to the controls when the cases with EM major were excluded. The increase in serum levels of IL-17A might indicate an active Th17 response in EM. Increased IL-6 is also supportive of this, since IL-6, along with increased TGF- $\beta$, promotes development of the Th17 cell line [15].

It has previously been established that EM is a Th1 cytokine-dominant reaction in which CD4+ T lymphocytes predominate [6]. CD4+ T lymphocytes were the majority of the inflammatory infiltrate in our patients, with lesser numbers of CD8+ cells. The characteristics of the infiltrate and cytokine milieu in SJS/TEN are different than EM and will not be discussed in this paper. The Th1-related cytokines IFN- $\gamma$ and IL-2 were found to be expressed lesional specimens of EM, whereas TNF- $\alpha$ has been found to be weakly expressed [16]. According to our knowledge, this is the first demonstration of the presence of IL-17-expressing cells in lesional samples of EM. The exact nature of these cells is yet to be determined. Interleukin 17A is among the main secretory products of Th17 cells, but other cell types such as $\gamma \delta$-T cells, CD8+ T cells, eosinophils, neutrophils and monocytes may also produce the cytokine [17]. We were not able to determine the exact nature of the cells expressing IL-17 in our study, but the possibility of them being Th17 cells is exciting enough to ponder on a wide array of possibilities.

The discovery of Th17 cells has damaged the orthodox view of the Th1/Th2 paradigm in immunology and it appears that this cell line is distinct from the mentioned subtypes of T helper cells. Although it is distinct, this cell line does not exist as the sole predominator of pathogenic $\mathrm{T}$ lymphocytes in a disease, but may be a cause, or the result of the ongoing process and co-exist with other $\mathrm{T}$ cell populations. In fact, Th17 cells and their cytokines may be important regulators of innate and adaptive immune responses in many Th1 and/or Th2-mediated diseases [18]. Th17 cells produce a group of cytokines including IL-17A, IL-17F, IL-21, IL-22, TNF- $\alpha$, and IFN- $\gamma$, in addition to IL-26 and chemokine ligand (CCL) 20 [19]. Interleukin 17 is important in the commencement and maintenance of autoimmune reactions and in production of proinflammatory cytokines, matrix metalloproteinases, neutrophils and eosinophils [20]. Either the presence of lesional IL-17-secreting cells or Th17-related serum cytokines has been demonstrated in various autoimmune and/or inflammatory diseases, such as psoriasis [21, 22], psoriatic arthritis [23], palmoplantar pustulosis [24], Behçet's disease [25], bullous pemphigoid [26], pemphigus vulgaris [26], systemic sclerosis [27] and vitiligo [28]. Besides their proposed proinflammatory role in various diseases, the Th17 cell line is crucial in protection against bacterial and fungal pathogens and is thought to be a part of host defences at the epithelial surfaces and potent in the recruitment of neutrophils [29, 30]. Their role in EM remains to be elucidated, though. A recent study has shown the presence of IL-17-secreting CD4+ lymphocytes in sera and blister fluid of patients with SJS/TEN and EM major. They reported that the proportion of IL-17-secreting CD4+ cells in the serum of SJS/TEN was higher than the controls. Although the difference was not significant when patients with EM major were compared to the controls, the number of patients in this study is quite few [31].

Th17 cells are activated CD $4^{+} \mathrm{T}$ cells. The orphan nuclear receptor ROR $\gamma \mathrm{t}$, a key transcription factor which induces the transcription of genes encoding IL-17A and IL$17 \mathrm{~F}$ in nadve $\mathrm{CD} 4^{+} \mathrm{T}$ helper cells. IL- 6 and TGF- $\beta$ are the cytokines known to induce this cell line [15]. It has been reported that HSV type 1 and type 2 are potent inducers of IL-6, and this phenomenon is augmented in the presence of IFN- $\gamma$ [32]. Transforming growth factor $\beta$ is expressed in lesions of EM [10]. Thus, the possibility comes to mind that Th17 may be formed de novo in EM. On the 
other hand, it has become clear that the Th17 lineage is not a single distinct lineage, but rather encompasses several $\mathrm{T}$ lymphocytes which all produce IL-17A, but possess varied effector functions. In fact, not all IL-17-producing T lymphocytes are pathogenic [19]. T-lymphocytes which are IFN- $\gamma+$ and IL-17+ originate from IL-17-producing Th17 cells, and are closely associated with pathogenicity [33]. It has previously been stated that $\mathrm{C}$. albicans and $\mathrm{S}$. aureus prime Th17 cells produce either IFN- $\gamma$ or IL-10 by eliciting different cytokines [34]. Interleukin $1 \beta$ and IL-2 are pro- and anti-inflammatory regulators of TH17 cells both at priming and in the effector phase [34]. Interleukin $1 \beta$ is a cytokine released from keratinocytes and has previously been linked to inflammatory skin disease. Th17 cells possess its receptors and secrete cytokines in response [35]. Instead of de novo formation, Th17 cells may be transformed into the more pathogenic IFN- $\gamma$-secreting types in EM. Although the differentiation of nadve CD4+ T cells has been viewed as an irreversible event and it is generally believed that fully differentiated Th1 cells have little or no plasticity to be redirected to become Th17 cells, it has been demonstrated that HSV-specific Th1 clones were still capable of producing IL-17 upon superantigen stimulation [36]. It might be possible that HSV-specific Th1 cells may be triggered by superantigen stimulation to produce IL-17. The IL-17-expressing cells in our samples may be of this type. A study supporting this idea has shown that superantigenic S. aureus stimulates IL-17 production from CD4+ $\mathrm{T}$ cells of adults with memory phenotypes but not from nadve CD4+ T cell phenotypes [37].

The association of HSV and Th17 cells has been studied in detail by Suryawanshi et al. in a study in which they studied a mouse model of corneal herpes infection leading to stromal keratitis, a chronic inflammatory reaction of the cornea to infection by herpes. Their data demonstrated that Th1 cells mainly initiate and orchestrate the lesions during the early stages, followed by the later entry of Th17 cells when the disease was at peak. The late entry of Th17 cells was partly explained by the delayed upregulation of IL- 6 and TGF- $\beta$, cytokines responsible for Th17 generation, as well as CCL20 expression in the cornea, a chemokine responsible for the migration of Th17 cells at the site of inflammation [38]. The authors commented that the body might sense the chronic persistence of antigen in HSV infections and as the need arises for stronger and more pathogenic immune response for the removal of such persistent antigen, the production of IL-17-producing cells or Th17 cells may be stimulated. This theory might explain the frequently detected presence of Th17 cells in autoimmune diseases and might be put to use in the pathogenesis of EM, where the chronic persistence of HSV DNA may presumably trigger a diversion of the Th1 responses to a Th17 direction. It is not known whether IL-17A is increased in the serum in between attacks in patients with recurrent EM, or whether the latent HSV infection itself is the trigger for the production of Th17 cells and IL-17A. In addition, the presence of other cytokines of the Th17 cell line, i.e. IL-21, 22 and 23 has not been investigated in EM. The presence of Th17 in an assisting role or an auto-reactive and possibly damaging (i.e. severe recurrent EM major) role is yet to be determined. The elucidation of the role of the Th17 axis in EM may shed light on the HSV and immune system interaction. A role for the Th17 axis in EM also brings to mind the possible therapeutic use of inhibitors of IL-17 and/or IL-23 in severe or recurrent cases of EM. At present, phase II and/or III trials of IL-17 inhibitors such as secukinumab, ixekizumab and brodalumab in the treatment of various diseases such as psoriasis, rheumatoid arthritis and Crohn's disease are being conducted. The effectiveness of these agents in the treatment of EM may be investigated [39].

The administration of small interfering RNA to mice with BD led to a decrease in the previously increased levels of IL-6 and improvement in BD symptoms. The serum levels of IL-17 also decreased (though the difference was not significant) and Foxp3+ regulatory $\mathrm{T}$ cells (Tregs) in these mice were more numerous compared to those in which IL-6 had not been inhibited [40]. Tregs, yet another cell line of the CD4+ T cell lineage, are a critical cell line in prevention of immune responses directed against self-antigens. It is known that Tregs efficiently suppress the effector functions of Th17 cells and it has been suggested that Treg-based therapies will be effective for Th17-mediated pathologies [19, 41]. It is necessary to question the role of Tregs and a possible deficiency in their responses in EM.

A limitation of this study was that serum cytokine levels were not reassessed after remission of lesions. Another limitation was that the exact nature of the IL-17A-secreting cells in the infiltrate could not be determined.

\section{Conclusions}

The discovery of specific cell types involved in EM and their links to HSV may advance our knowledge on important links between HSV and the immune system. As more knowledge on the subject is attained, novel therapies targeting key steps may be developed.

This project was supported by grants from the Dicle University Scientific Projects Commission (grant number: 12-TF-130).

The authors declare no conflict of interest.

\section{References}

1. Spandau U, Bröcker EB, Kämpgen E, Gillitzer R (2002): CC and CXC chemokines are differentially expressed in erythema multiforme in vivo. Arch Dermatol 138: 1027-1033. 
2. Darragh TM, Egbert BM, Berger TG, Yen TS (1991): Identification of herpes simplex virus DNA in lesions of erythema multiforme by the polymerase chain reaction. J Am Acad Dermatol 24: 23-26.

3. Sokumbi O, Wetter DA (2012): Clinical features, diagnosis, and treatment of erythema multiforme: a review for the practicing dermatologist. Int J Dermatol 51: 889-902.

4. LeBoit PE (1993): Interface dermatitis. How specific are its histopathologic features? Arch Dermatol 129: 1324-1328.

5. Joshi R (2013): Interface dermatitis. Indian J Dermatol Venereol Leprol 79: 349-359.

6. Margolis RJ, Tonnesen MG, Harrist TJ, (1983): Lymphocyte subsets and Langerhans cells/indeterminate cells in erythema multiforme. J Invest Dermatol 81: 403-406.

7. Imafuku S, Kokuba H, Aurelian L, Burnett J (1997): Expression of herpes simplex virus DNA fragments located in epidermal keratinocytes and germinative cells is associated with the development of erythema multiforme lesions. J Invest Dermatol 109: 550-556.

8. Ono F, Sharma BK, Smith CC (2005): CD34+ cells in the peripheral blood transport herpes simplex virus DNA fragments to the skin of patients with erythema multiforme (HAEM). J Invest Dermatol 124: 1215-1224.

9. Aurelian L, Kokuba H, Burnett JW (1998): Understanding the pathogenesis of HSV-associated erythema multiforme. Dermatology 197: 219-222.

10. Kokuba H, Aurelian L, Burnett J (1999): Herpes simplex virus associated erythema multiforme (HAEM) is mechanistically distinct from drug-induced erythema multiforme: interferon-gamma is expressed in HAEM lesions and tumor necrosis factor-alpha in drug-induced erythema multiforme lesions. J Invest Dermatol 113: 808-815.

11. Gober MD, Laing JM, Burnett JW, Aurelian L (2007): The Herpes simplex virus gene Pol expressed in herpes-associated erythema multiforme lesions upregulates/activates SP1 and inflammatory cytokines. Dermatology 215: 97-106.

12. Giordano CN, Sinha AA (2012): Cytokine networks in Pemphigus vulgaris: An integrated viewpoint. Autoimmunity 45: 427-439.

13. Chodorowska G, Czelej D, Niewiedzioł M. Interleukin-2 and its soluble receptor in selected drug-induced cutaneous reactions. Ann Univ Mariae Curie Sklodowska Med 2003; 58: 7-13.

14. Chodorowska G, Czelej D, Krasowska D, Pietrzak A. Plasma activity of interleukin-10 in drug-induced cutaneous reactions. Ann Univ Mariae Curie Sklodowska Med 2003; 58: 317-321.

15. Kim JO, Cha HR, Kim ED, Kweon MN. Pathological effect of IL-17A-producing TCR $\gamma \delta(+)$ T cells in mouse genital mucosa against HSV-2 infection. Immunol Lett 2012; 147 : 34-40.

16. Caproni M, Torchia D, Schincaglia E, et al. (2006): Expression of cytokines and chemokine receptors in the cutaneous lesions of erythema multiforme and Stevens-Johnson syndrome/toxic epidermal necrolysis. Br J Dermatol 155: 722728.

17. Lewis BJ, Rajpara S, Haggart AM, et al. (2013): Predominance of activated, clonally expanded $\mathrm{T}$ helper type 17 cells within the CD4+ T cell population in psoriatic lesions. Clin Exp Immunol 173: 38-46.

18. Ghoreschi K, Laurence A, Yang XP,(2011): T helper 17 cell heterogeneity and pathogenicity in autoimmune disease. Trends Immunol 32: 395-401.
19. Bedoya SK, Lam B, Lau K, Larkin J 3rd (2013): Th17 cells in immunity and autoimmunity. Clin Dev Immunol 2013: 986789.

20. Harrington LE, Hatton RD, Mangan PR, et al. (2005): Interleukin 17-producing CD4+ effector T cells develop via a lineage distinct from the $\mathrm{T}$ helper type 1 and 2 lineages. Nat Immunol 6: 1123-1132.

21. Balato A, Schiattarella M, Di Caprio R,et al. (2014): Effects of adalimumab therapy in adult subjects with moderate-to-severe psoriasis on Th17 pathway. J Eur Acad Dermatol Venereol 28: 1016-1024.

22. Kagami S, Rizzo HL, Lee JJ, et al. (2010): Circulating Th17, Th22, and Th1 cells are increased in psoriasis. J Invest Dermatol 130: 1373-1383.

23. Benham H, Norris P, Goodall J, et al. (2013): Th17 and Th22 cells in psoriatic arthritis and psoriasis. Arthritis Res Ther 15: R136.

24. Hagforsen E, Hedstrand H, Nyberg F, Michaëlsson G (2010): Novel findings of Langerhans cells and interleukin-17 expression in relation to the acrosyringium and pustule in palmoplantar pustulosis. Br J Dermatol 163: 572-579.

25. Na SY, Park MJ, Park S, Lee ES (2013): Up-regulation of Th17 and related cytokines in Behçet's disease corresponding to disease activity. Clin Exp Rheumatol 31: 32-40.

26. Arakawa M, Dainichi T, Ishii N, et al. (2011): Lesional Th17 cells and regulatory T cells in bullous pemphigoid. Exp Dermatol 20: 1022-1024.

27. Truchetet ME, Brembilla NC, Montanari E, et al. (2014): Interleukin-17A+ cell counts are increased in systemic sclerosis skin and their number is inversely correlated with the extent of skin involvement. Arthritis Rheum 65: 1347-1356.

28. Kotobuki Y, Tanemura A, Yang L, et al. (2012): Dysregulation of melanocyte function by Th17-related cytokines: significance of Th17 cell infiltration in autoimmune vitiligo vulgaris. Pigment Cell Melanoma Res 25: 219-230.

29. Sakai A, Sugawara Y, Kuroishi T, et al. (2008): Identification of IL-18 and Th17 cells in salivary glands of patients with Sjögren's syndrome, and amplification of IL-17-mediated secretion of inflammatory cytokines from salivary gland cells by IL-18. J Immunol 181: 2898-2906.

30. Hammerich L, Heymann F, Tacke F (2011): Role of IL-17 and Th17 cells in liver diseases. Clin Dev Immunol 2011: 345803.

31. Teraki Y, Kawabe M, Izaki S (2013): Possible role of TH17 cells in the pathogenesis of Stevens-Johnson syndrome and toxic epidermal necrolysis. J Allergy Clin Immunol 131: 907909.

32. Paludan SR (2001): Requirements for the induction of interleukin- 6 by herpes simplex virus-infected leukocytes. J Virol 75: 8008-8015.

33. Hirota K, Duarte JH, Veldhoen M, et al. (2011):. Fate mapping of IL-17-producing T cells in inflammatory responses. Nat Immunol 12: 255-263.

34. Zielinski CE, Mele F, Aschenbrenner D, et al. (2012):. Pathogen-induced human TH17 cells produce IFN- $\gamma$ or IL-10 and are regulated by IL-1 $\beta$. Nature 484: 514-518.

35. Cho KA, Suh JW, Lee KH, et al. (2012): IL-17 and IL-22 enhance skin inflammation by stimulating the secretion of IL-1 $\beta$ by keratinocytes via the ROS-NLRP3-caspase-1 pathway. Int Immunol 24: 147-158.

36. Yomogida K, Chou YK, Chu CQ (2013): Superantigens induce IL-17 production from polarized Th1 clones. Cytokine 63: 6-9. 
37. Islander U, Andersson A, Lindberg E, et al. (2010): Superantigenic Staphylococcus aureus stimulates production of interleukin-17 from memory but not naive T cells. Infect Immun 78: $381-386$

38. Suryawanshi A, Veiga-Parga T, Rajasagi NK, et al. (2011): Role of IL-17 and Th17 cells in herpes simplex virus-induced corneal immunopathology. J Immunol 187: 1919-1930.

39. Miossec P, Kolls JK (2012): Targeting IL-17 and TH17 cells in chronic inflammation. Nat Rev Drug Discov 11: 763-776.

40. Shim J, Byun HO, Lee YD, et al. (2009): Interleukin-6 small interfering RNA improved the herpes simplex virus-induced systemic inflammation in vivo Behcet's disease-like mouse model. Gene Ther 16: 415-425.

41. Crome SQ, Clive B, Wang AY, et al. (2010): Inflammatory effects of ex vivo human Th17 cells are suppressed by regulatory T cells. J Immunol 185: 3199-3208. 